\title{
The Impact of a Corticotectal Impulse on the Awake Superior Colliculus
}

\author{
Yulia Bereshpolova, Carl R. Stoelzel, Alexander G. Gusev, Tatiana Bezdudnaya, and Harvey A. Swadlow \\ Department of Psychology, University of Connecticut, Storrs, Connecticut 06269
}

Corticotectal (CTect) neurons of layer 5 are large and prominent elements of mammalian visual cortex, with thick apical dendrites that ascend to layer 1, "intrinsically bursting" membrane properties, and fast-conducting descending axons that terminate in multiple subcortical domains. These neurons comprise a major output pathway of primary visual cortex, but virtually nothing is known about the synaptic influence of single CTect impulses on the superior colliculus (SC). Here, we examine the distribution of monosynaptic currents generated in the superficial SC by spontaneous impulses of single CTect neurons. We do this by recording the spikes of CTect neurons and the field potentials that they generate through the depths of the SC. Methods of spike-triggered averaging and current source density analysis are then applied to these data. We show, in fully awake rabbits, that single CTect impulses generate potent, fast-rising monosynaptic currents in the SC similar to those generated in sensory cortex by specific thalamic afferents. These currents are focal in depth, precisely retinotopic, and highly dependent on the conduction velocity of the CTect axon. Moreover, we show that CTect synapses, like thalamocortical synapses, suffer a chronic state of depression in awake subjects that is modulated by preceding interspike interval. However, CTect neurons generated few "bursts," and postsynaptic responses in the SC were not significantly influenced by a shift from alert to an inattentive state (indicated by hippocampal EEG). Together, our results suggest that single CTect neurons may resemble thalamocortical neurons in their ability to serve as potent "drivers" of postsynaptic targets.

Key words: superior colliculus; visual cortex; corticotectal; synaptic depression; intrinsically bursting; awake recording

\section{Introduction}

Layer 5 of primary sensory cortices contains a prominent subpopulation of large pyramidal neurons with thick apical dendrites that ascend to layer 1 (Hallman et al., 1988; Hubner et al., 1990; White, 1990). These cells possess fast-conducting axons that branch to enervate multiple subcortical targets, and intracortical collaterals that may terminate several millimeters from the soma (Swadlow and Weyand, 1981; Keizer et al., 1987; Hallman et al., 1988). Notably, these neurons display "intrinsically bursting” membrane properties (Connors et al., 1982; McCormick et al., 1985) and may mediate the intracortical transmission of oscillatory activity (Chagnac-Amitai and Connors, 1989). In primary visual cortex (V1), the great majority of corticotectal (CTect) neurons are members of this distinctive subpopulation of pyramidal neurons (Kasper et al., 1994; Rumberger et al., 1998; Tsiola et al., 2003). In contrast, layer 5 corticocortical neurons have smaller somata, and thinner and shorter apical dendrites, and do not display intrinsic bursting (Hallman et al., 1988; Hubner et al., 1990; Kasper et al., 1994).

Despite the prominence of this distinctive population, there

Received April 1, 2005; revised Jan. 10, 2006; accepted Jan. 14, 2006.

This work was supported by National Science Foundation Grant IOB-0445583 and National Institute of Mental Health Grant MH-64024. Multichannel probes were provided by the University of Michigan Center for Neural Communication Technology. We thank Victor Serdyukov for expert hardware and software development.

Correspondence should be addressed to Harvey A. Swadlow, Department of Psychology (U-1020), University of Connecticut, Storrs, CT 06269. E-mail: harvey.swadlow@uconn.edu.

DOI:10.1523/JNEUROSCI.4402-05.2006

Copyright $\odot 2006$ Society for Neuroscience $\quad$ 0270-6474/06/262250-10\$15.00/0 are no available data concerning the synaptic effects of single CTect neurons on the superior colliculus (SC). Thus, there have been no in vitro paired recordings, or in vivo crosscorrelation studies of the impact of single CTect neurons on SC target neurons. Moreover, there have been no intracellular labeling studies of CTect axons. We do not know, therefore, whether single CTect neurons generate a dense or sparse terminal arborization within the SC, the retinotopic precision of single-axon arborizations, or the postsynaptic impact generated by single CTect neurons.

$\mathrm{CTect}$ neurons of $\mathrm{V} 1$ terminate in superficial, retinal recipient layers of the SC in a roughly topographic manner (McIlwain, 1973; Hollander and Schonitzer, 1983; Harting et al., 1992). Moreover, many superficial SC neurons respond to both retinal and CTect stimulation (Berson, 1988), and some receptive field properties are lost or modified after V1 inactivation (Rizzolatti et al., 1970; Rosenquist and Palmer, 1971; Ogasawara et al., 1984). The mechanism of this influence, however, is still unknown (Hashemi-Nezhad et al., 2003).

The synaptic impact of single neurons on postsynaptic targets has generally been explored using paired intracellular recordings in vitro, or using cross-correlation methods in vivo. In such experiments, the influence of a single presynaptic neuron on a single postsynaptic target neuron is usually investigated. Here, we use a different approach to gain a broader, more global view of the synaptic impact of single cortical neurons on distant targets. We do this by recording the spikes of CTect neurons and the field potentials that they generate through the depths of the SC (Swad- 

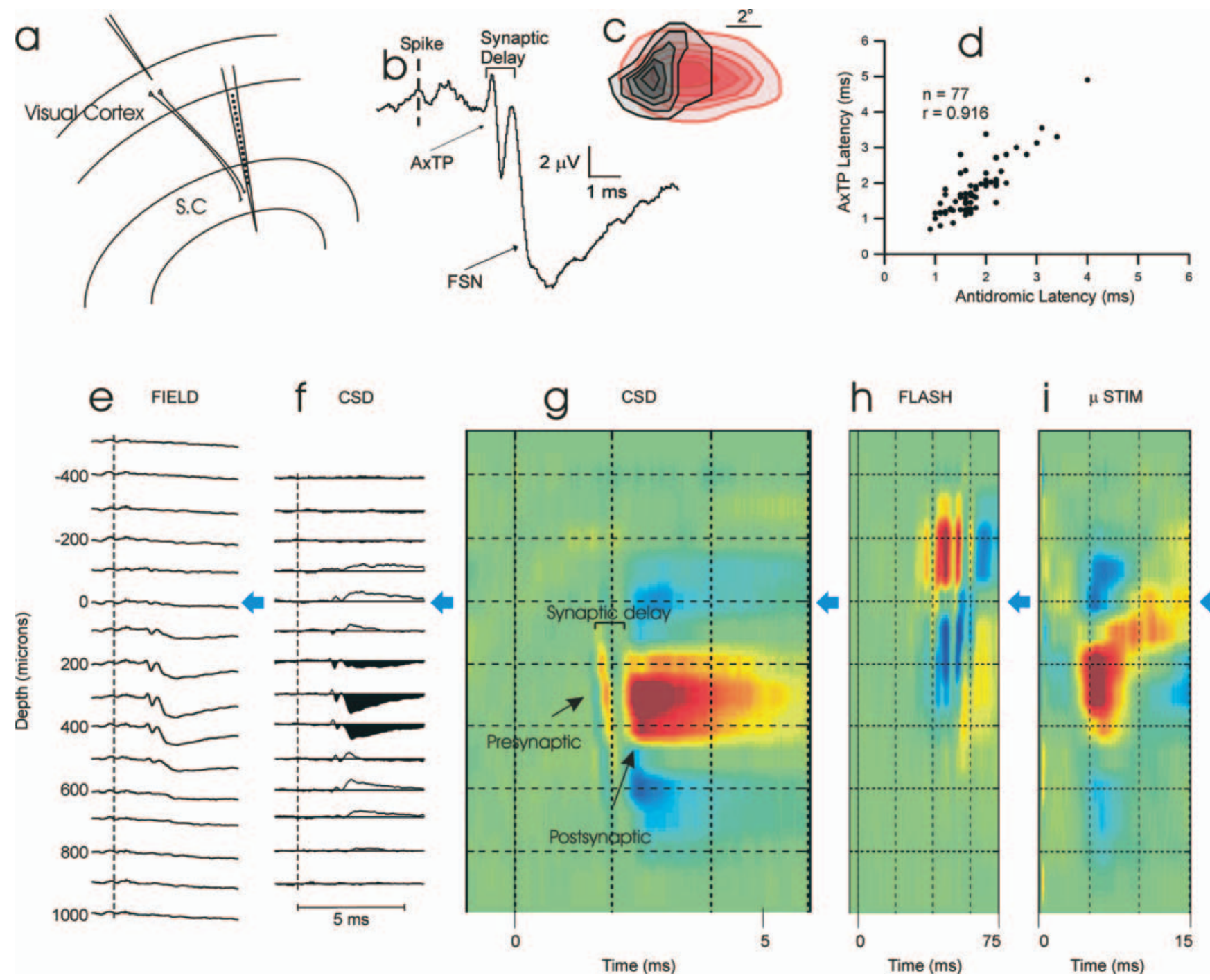

Figure 1. a, Schematic illustration of recording situation. A microelectrode records from a CTect neuron in V1 and spike-triggered field potentials are recorded using a 16 -channel probe (100 $\mu \mathrm{m}$ vertical site separation) located within the aligned region of the SC. A stimulating electrode (data not shown), located 1-2 mm from the SC probe site, was used to antidromically identify (Tect neurons. $\boldsymbol{b}$, A spike-triggered field potential trace, generated by the spontaneous spikes of a (Tect neuron, at a SC recording site $\sim 650 \mu \mathrm{m}$ beneath the surface of the SC (trace 9 from $\boldsymbol{e}$ below), near the peak of the spike-triggered presynaptic and postsynaptic potentials. The vertical dashed line represents the time of the (Tect action potential. The AxTP and the postsynaptic current sink (FSN) are indicated by black arrows, and the synaptic delay (between the onset of these two potentials) is also shown. c, Receptive field alignment of the (Tect neuron (black) and the recording site shown in $\boldsymbol{b}$. $\boldsymbol{d}$, Relationship between the latency of the AxTP (after the CTect spike) and the antidromic latency of the (Tect neuron to electrical stimulation of the SC. Depth profiles of spike-triggered field potentials $(\boldsymbol{e})$ and the CSD analysis of these potentials $(\boldsymbol{f})$ generated by the same CTect neuron. $\boldsymbol{g}$, Colorized version of the CSD analysis, with current sinks (inward, excitatory currents) indicated in red and sources indicated in blue. $\boldsymbol{h}$, CSD profile generated by flashing visual stimulus using the same SC probe. The horizontal blue arrows seen here and in subsequent figures mark the reversal point in the CSD generated from the flashing stimulus.i, CSD profile generated by microstimulation ( $\mu$ STIM) (10 $\mu \mathrm{A})$ within layer 5 , near the site of the CTect neuron shown in $\boldsymbol{e}-\boldsymbol{g}$. The amplitude gains for colorization in $\boldsymbol{h}$ and $\boldsymbol{i}$ were $1 / 30$ and $1 / 20$ that in $\boldsymbol{g}$, respectively. Note the different time bases of $\boldsymbol{g}, \boldsymbol{h}$, and $\boldsymbol{i}$.

low et al., 2002) and applying methods of spike-triggered averaging (Kirkwood, 1995; Swadlow and Gusev, 2000) and current source density (CSD) analysis (Haberly and Shepherd, 1973; Freeman and Nicholson, 1975; Buzsaki and Kandel, 1998) to these data. This allowed us to examine, in fully awake rabbits, the vertical distribution of monosynaptic currents generated in the superficial layers of the SC by spontaneous impulses of single CTect neurons, and the temporal dynamics of these synaptic currents.

\section{Materials and Methods}

Figure $1 a$ illustrates our experimental methods, used in awake, adult, Dutch-belted rabbits. Our goal was to record extracellular "spontaneous" spikes from CTect neurons in primary visual cortex (V1) and spike- triggered field potentials through the depths of the retinotopically aligned region of the superficial SC. Initial surgery was performed under anesthesia using aseptic procedures, and subsequent recordings were obtained from awake subjects using procedures approved by the Institutional Animal Care and Use Committee at the University of Connecticut. Methods to ensure the comfort of our subjects have been described previously (Swadlow, 1995; Swadlow et al., 2002). Rabbits were held snugly within a stocking and placed on a foam pad. A steel bar on the head was fastened to a restraining device in a manner that minimized stress on the neck. Hippocampal EEG activity was monitored via recording electrodes placed above and below the CA1 layer.

Microelectrodes for recording from CTect neurons and for preliminary receptive field mapping were constructed from quartz-insulated, platinum-tungsten filaments, pulled to a taper and sharpened to a fine 
tip (Reitboeck, 1983). SC recordings were obtained using 16-channel silicone probes (University of Michigan Center for Neural Communication Technology, Ann Arbor, MI). Probe sites were separated vertically by $100 \mu \mathrm{m}$ and had surface areas of 400 or $700 \mu \mathrm{m}^{2}$ and impedances of 0.3-0.8 $\mathrm{M} \Omega$. Recordings were obtained from monocular regions of $\mathrm{V} 1$ and the SC that lie within $10^{\circ}$ of the visual streak (the visual horizon) (Levick, 1967).

The success of these experiments depended on achieving retinotopic alignment of two types: (1) between the V1 and SC recording sites and (2) among the recording sites located at various depths within the SC. This latter type of alignment was the most difficult to achieve because of the considerable curvature of the SC. To do this, the SC was first mapped using a single microelectrode, and the angle of the electrode was adjusted until the receptive fields at various depths indicated that the electrode was approximately normal to the SC surface. The mapping electrode was then replaced by the 16-channel probe, which was lowered until the deepest site was $\sim 1.2 \mathrm{~mm}$ beneath the surface of the SC. Simultaneous receptive field maps (below) were obtained from each of the probe sites to estimate depth within the SC and to assure that the probe sites were in alignment. The probe was then cemented into place using acrylic cement. A plastic cap protected the probe between recording sessions. Probes retained good recording characteristics and stable impedances for several days, but impedance values would then begin to rise. Microstimulation pulses delivered to probe sites $(10 \mu \mathrm{A}$; cathodal; $30 \mathrm{~Hz}$ for $3 \mathrm{~s}$; then repeating these parameters but with reversed polarity) considerably increased the useful lifetime of the probes by returning impedances to near their original values. It was desirable to record from a single probe as long as possible so that the impact of many topographically aligned (and nonaligned) CTect neurons could be assessed and compared.

Once the probe was cemented into place, the skull was thinned (to a thickness of $\sim 0.4 \mathrm{~mm})$ over the region of primary visual cortex $(\sim 3 \times 4$ $\mathrm{mm}$ ) in retinotopic alignment with the SC probe. Recordings were obtained, during subsequent recording sessions, through small holes drilled through this thinned bone. The dura was left intact. CTect neurons were identified by antidromic activation via stimulating electrodes placed in the SC, $\sim 1-2 \mathrm{~mm}$ rostral of the multichannel probe (on the estimated trajectory of the CTect axons).

Spike data were acquired from CTect neurons and from each of the 16 SC sites using a Plexon (Dallas, TX) data acquisition system. Spike data from the SC probe sites generally consisted of low-amplitude multiunit activity, used for plotting receptive fields. Field potentials were also recorded at each of the $16 \mathrm{SC}$ probe sites, filtered at $2 \mathrm{~Hz}$ to $1.9 \mathrm{kHz}$ (half-amplitude), and sampled continuously at 5 or $10 \mathrm{kHz}$. Spiketriggered averages of the cortical field activity were generated from the spontaneous impulse activity of CTect neurons as follows: for the "control" record, we used all of the spikes in the data set except those that occurred within $5 \mathrm{~ms}$ of another spike. We eliminated CTect spikes with short $(<5 \mathrm{~ms})$ interspike intervals to avoid generating compound averages from any high-frequency spikes. When examining the effects of preceding interspike interval on the spike-triggered potentials, we analyzed only those CTect spikes that (1) were preceded by interspike intervals of a given value and (2) were not followed by another spike within $5 \mathrm{~ms}$.

Receptive fields were plotted simultaneously for the CTect neurons under study and at each of the SC probe sites. Receptive field mapping was accomplished using sparse noise stimulation consisting of small flashing light and dark stimuli presented on a computer monitor. Stimuli consisted of high-contrast squares $\left(0.5-2^{\circ}\right)$ presented pseudorandomly, in a grid of $30 \times 22$. Each square was presented for either 10 or $20 \mathrm{~ms}$. The size of each grid space was $1^{\circ}$, and the size of the squares was either $1^{\circ}$ or $2^{\circ}$. Receptive fields were constructed using methods of reverse correlation (Jones and Palmer, 1987) and smoothed, and contour lines were fitted using bilinear interpolation. Receptive field size was calculated as the number of grid spaces included within the $30 \%$ contour line of the maximum response. We have implemented a real-time version of this procedure that enables the sensitive receptive fields in superficial SC to be mapped very quickly. In a lighted environment, the eye of the rabbit is remarkably stable, usually remaining within a region of $\pm 0.5^{\circ}$ for periods of several minutes (Collewijn, 1977; Swadlow and Weyand, 1987). Because SC receptive fields are much larger than this (the smaller SC fields are $\sim 3 \times 2^{\circ}$ ), and because they can usually be generated within $30 \mathrm{~s}$ of stimulation onset, eye movements did not present a problem. During the several days in which each probe was studied, we plotted the receptive fields on each of the probe sites in excess of 100 times, and it was obvious when an eye movement had occurred (the same "smearing" of the receptive field could be seen at each of the probe sites). SC and cortical receptive fields were acquired and plotted simultaneously to examine retinotopic alignment of the cortical and SC sites.

The degree of receptive field overlap between CTect and SC sites was calculated by comparing the receptive fields of the CTect neuron with the receptive field of the multiunit SC response recorded on the SC probe site that was $\sim 200 \mu \mathrm{m}$ below the reversal point of the flash-evoked response within the SC. This site corresponds to the depth of the predominant postsynaptic impact of CTect input to the SC (see Results). The degree of overlap was given by the proportion of the CTect receptive field that was encompassed by the SC receptive field (30\% contour lines for both fields).

Depth profiles of the synaptic impact of CTect impulses were generated using methods previously described (Swadlow and Gusev, 2000; Swadlow et al., 2002). Spike-triggered averages of the cortical field activity were generated from the spontaneous impulse activity of CTect neurons. Postsynaptic responses were considered to be reliable only when the following three conditions were met: (1) they were preceded by a clear presynaptic component of $\geq 1 \mu \mathrm{V}$ in amplitude (the axon terminal component) (see below), (2) they followed this axon terminal component by $<1 \mathrm{~ms}$ and consisted of a sharp negativity in the spike-triggered record, and (3) this same sequence of events resulted from at least two separate trains of CTect spikes.

CSD profiles were generated from the field profiles according to the method described by Freeman and Nicholson (1975). First, we duplicated the uppermost and lowermost field trace (Borroni et al., 1991), which converted our 16 recording channels to a total of 18 channels. Next, we smoothed (Freeman et al., 1975) to reduce high spatialfrequency noise components. This eliminated 2 of the 18 traces.

$$
\text { Smoothing: } \bar{\phi}(r)=\frac{1}{4}(\phi(r+h)+2 \phi(r)+\phi(r-h)),
$$

where $\phi$ is the field potentials, $r$ is the coordinate perpendicular to the layers, and $h$ is the sampling interval $(100 \mu \mathrm{m})$.

Next, we calculated the second derivative, and this yielded a total of 14 traces.

$$
\text { Second derivative: } D=\frac{1}{h^{2}}(\bar{\phi}(r+h)-2 \bar{\phi}(r)+\bar{\phi}(r-h))
$$

In the CSD profiles, current sinks are indicated by downward deflections and sources by upward deflections. To facilitate visualization of CSD profiles, we generated color image plots. These were plotted by linear interpolation along the depth axis, red and blue representing current sinks and sources, respectively. Green is approximately zero.

When the analysis of a CTect neuron was complete, we examined depth profiles within the SC that were generated by (1) brief, full-field visual flashes and (2) microstimulation pulses at, or very close $(<100$ $\mu \mathrm{m})$ to, the CTect recording site within layer $5(10 \mu \mathrm{A}$; cathodal; $0.3 \mathrm{~ms}$ duration; 1 or $2 / \mathrm{s}$ ). These depth profiles provided a useful depth reference for the low-amplitude field potentials and synaptic currents generated by each of the CTect neurons studied.

\section{Results}

\section{The impact of a CTect impulse on the SC}

In two rabbits, we examined the synaptic impact of $136 \mathrm{CTect}$ neurons that each showed some degree of receptive field overlap with receptive fields on our SC probe. For 90 of these neurons, the receptive fields of the CTect neuron and the SC site overlapped by $>50 \%$, and the remainder overlapped by $1-50 \%$. An additional 13 CTect neurons showed no receptive field overlap, but were misaligned by less than one receptive field diameter. Most (77 of 136) CTect neurons that showed some degree of receptive field 
overlap with our SC sites showed a reliable postsynaptic impact. Figure $1 b$ shows a spike-triggered average field potential generated by the impulses of a CTect neuron at a recording site located $\sim 650 \mu \mathrm{m}$ beneath the surface of the aligned region of the SC (Fig. $1 c$ shows retinotopic alignment of this SC probe site with the receptive field of the CTect neuron).

The response profile generated by this CTect neuron, and by nearly all of the CTect neurons that we studied, could be readily divided into two components: (1) a short-latency, shortduration, biphasic or triphasic response (usually initially positive-going) reflecting invasion of the presynaptic impulse into the axon terminal arborization [the axon terminal potential (AxTP)] (Fig. 1b, top arrow). The presynaptic nature of the AxTP is supported by the short duration of this response, the rapid rise time (mean, $0.17 \mathrm{~ms} ; 10-90 \%$ ), the close agreement between the latency of the AxTP and the antidromic latency of the CTect neuron to stimulation of the SC (Fig. $1 d)(r=0.92 ; n=77)$, and the invariant amplitude of this response in the face of varying preceding interspike interval (see Fig. 4c). (2) A negative-going potential followed the AxTP by $<1 \mathrm{~ms}$ and is believed to reflect a focal synaptic depolarization [the focal synaptic negative potential (FSN)] (Swadlow and Gusev, 2000; Swadlow et al., 2002) (Fig. $1 b$, bottom arrow). The FSN was fast-rising (mean, $0.47 \mathrm{~ms} ; 10-$ $90 \%)$ and reached a mean maximal amplitude of $5.77 \mu \mathrm{V}( \pm 3.4$ $\mu \mathrm{V}$; range, $0.8-14.7 \mu \mathrm{V})$. Figure $1 e$ shows the depth profile of the field potentials generated by this CTect neuron, and Figure $1 f$ shows the CSD of these profiles. A colorized version of the CSD profile is shown in Figure $1 g$, in which red reflects inward currents (sinks) and blue reflects current sources. For a given CTect neuron, the spike-triggered CSD profiles were very stable over time. This neuron was studied for $>5 \mathrm{~h}$, and the CSD profile taken during the initial $0.5 \mathrm{~h}$ period shown here was nearly indistinguishable from those taken during the later recordings.

The above values for the amplitude and rise times of the FSN and AxTP were taken from 66 CTect neurons (1) in which receptive fields overlapped by $>50 \%$ with SC fields and (2) that showed some reliable postsynaptic impact. As described in $\mathrm{Ma}-$ terials and Methods, to conclude that a CTect neuron generated a reliable postsynaptic response, we required that an unambiguous $(>1 \mu \mathrm{V})$ presynaptic response also be present (the AxTP). We rejected three cases from our data set in which a postsynaptic component seemed to be present, but no presynaptic component was visible. It is worth noting that the amplitude of the FSN generated by some CTect neurons can be considerably larger than the values reported here. In initial experiments, we recorded within the SC using a single low-impedance recording microelectrode (rather than the 16-channel probe). Most of the CTect neurons that we studied using this method generated similar values to those reported here, but one CTect neuron generated an FSN with an amplitude of $27.3 \mu \mathrm{V}$. We did not include these recordings in our data set, because we could not be sure that all of them were obtained at the optimal depth within the SC, and we began using the 16-channel recording probe to eliminate this problem.

\section{The depth of monosynaptic currents generated in the SC by a CTect neuron}

We also examined, at each SC probe site, the vertical distribution of field potentials and currents elicited by (1) a brief, full-field visual (flash) stimulus (Fig. 1h) and by (2) microstimulation within layer 5, near the site of each of the CTect neurons (see Materials and Methods) (Fig. 1i). The depth profiles generated by these measures provided a useful depth reference for the field potentials and synaptic currents generated by each of the CTect neurons studied.

Microlesions made at the reversal point of the visual evoked CSD were located 205-500 $\mu \mathrm{m}$ (mean, $354 \mu \mathrm{m} ; n=11$ ) beneath the surface of the SC (corresponding to the mid-upper portion of the striatum grisium superficiale) (Hofbauer and Hollander, 1986), and most CTect neurons generated peak synaptic currents 200-300 $\mu \mathrm{m}$ deeper than this reversal point. There were, however, differences among CTect neurons in the depth of the synaptic impact. Figure $2 a-c$ shows the depth profiles of currents generated on a single SC probe by three neighboring CTect neurons that generated focal synaptic currents with distinct depth profiles and latencies. The horizontal blue arrow shows the reversal point of the flash-evoked response on this probe site (Fig. $2 d$ ). Figure $2 e$ (black lines) shows the depth distribution of the peak synaptic current sinks generated by all of the CTect neurons that we studied, relative to the reversal point of the visual stimulus.

Current sinks generated by microstimulation of layer 5 reached peak intensities at depths that were very similar to those generated by the impulses of most CTect neurons (Fig. 1i). Figure $2 e$ (dashed red lines) shows the distribution of current peaks generated by microstimulation pulses, relative to the reversal depth of the flash-evoked responses. In each case, peak amplitudes were generated either two or three recording sites (200-300 $\mu \mathrm{m})$ beneath the reversal point of the flash-evoked response.

\section{Factors governing the strength and probability with which a CTect neuron impacts the SC}

Seventy-seven of the 136 CTect neurons showing some degree of receptive field overlap with our SC sites showed a reliable postsynaptic impact. Figure $3 a$ shows the relationship between the degree of receptive field overlap and the amplitude and probability of observing a reliable postsynaptic response of CTect neurons (the probability of a reliable postsynaptic response refers to the proportion of CTect neurons that yielded a reliable response, as defined in Materials and Methods). Because of the strong relationship between axonal conduction time and CTect impact (see below), for this analysis we included only those CTect neurons with axonal conduction times of $<2 \mathrm{~ms}$. Whereas $>80 \%$ of CTect neurons with receptive field overlap of $>40 \%$ generated a reliable monosynaptic impact on the SC, none of those with overlaps of $<20 \%$ did so. The degree of receptive field overlap between CTect and SC recording sites was significantly related to both the amplitude of the postsynaptic response $(p<0.001)$ and the probability of detecting a reliable response $(p<0.005)$.

Conduction velocity of the CTect axon was another factor that was strongly related to the postsynaptic impact of a CTect neuron. Conduction time along CTect axon (based on antidromic latency) varied from 0.9 to $7.6 \mathrm{~ms}$. Figure $3 b$ shows the relationship between the antidromic latency of the CTect axon and the amplitude and probability of monosynaptic responses of CTect neurons. Because of the strong relationship between receptive field overlap and CTect impact (above), for this analysis we included only those CTect neurons with receptive field overlap at the SC sites of $>50 \%$. Whereas $>80 \%$ of CTect neurons with antidromic latencies of $<2 \mathrm{~ms}$ generated a reliable monosynaptic impact on the SC, none with conduction times of $>5 \mathrm{~ms}$ did so. Conduction time along the CTect axon was strongly related to both the amplitude of the postsynaptic response $(p<0.001)$ and to the probability of detecting a reliable response $(p<0.025)$. 

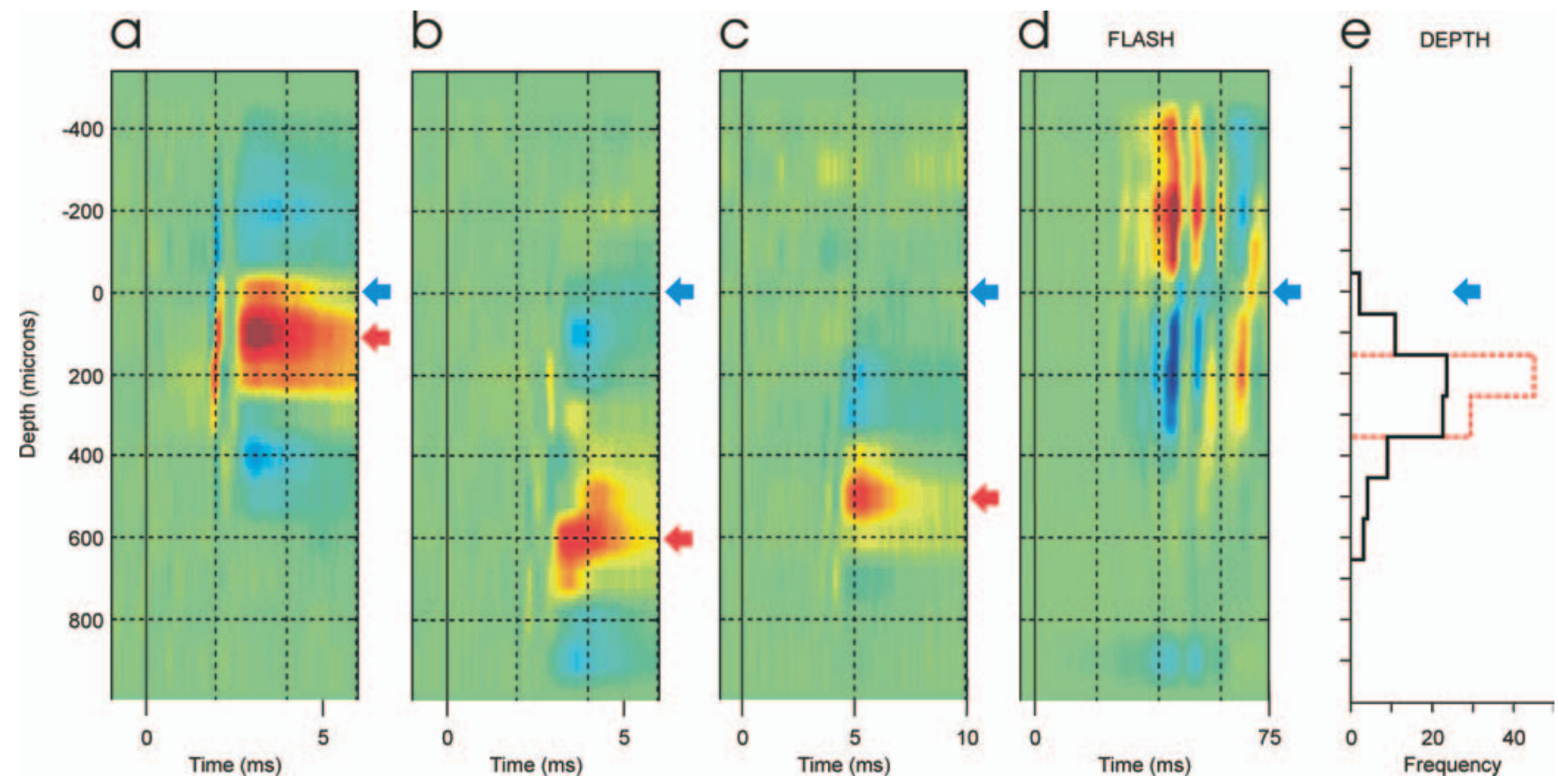

Figure 2. $\boldsymbol{a}-\boldsymbol{c}$, Colorized CSD analyses of spike-triggered field potentials generated on a single 16-channel recording probe in the SC by the spontaneous spikes of three neighboring CTect neurons. The depth of the reversal point in the flash-evoked response $(\boldsymbol{d})$ is indicated by the blue arrows, and the depths of the peak synaptic currents $(\boldsymbol{a}-\boldsymbol{c})$ are indicated by the red arrows. The gains in $\boldsymbol{a}$ - c are 30 times greater than that in $\boldsymbol{d}$. $\boldsymbol{e}$, The solid lines indicate the depth, relative to the reversal point of the flashing visual stimulus, of the peak synaptic current sink generated by all of the CTect neurons that we studied. The red dashed lines indicate the distribution of current peaks generated by microstimulation pulses delivered to layer 5 , relative to the reversal depth of the flash-evoked responses.

\section{Dynamics of postsynaptic currents generated by a CTect impulse}

The postsynaptic impact of a CTect impulse depended strongly on the duration of the preceding interspike interval. Figure 4 shows the spike-triggered CSD depth profiles of a CTect neuron that generated a powerful current sink in the superficial SC. More than 19,000 spikes were generated by this neuron in $\sim 1.5 \mathrm{~h}$ of study, but in Figure $4 a$, only those spikes with short (5-25 ms) preceding interspike intervals were used to generate the depth profiles. Figure $4 b$ shows the CSD profile for this same CTect neuron, but here we selected only impulses with long preceding interspike intervals $(>450 \mathrm{~ms})$ to generate the depth profiles. Note that, although the amplitude of the postsynaptic current sink is substantially larger in Figure $4 b$ than in Figure $4 a$, the amplitude of the presynaptic (axon terminal) potential (arrows in colorized version) is approximately the same. Figure $4 c$ shows, at various preceding interspike intervals, the spike-triggered field potential on channel 11 of our 16-channel probe. Whereas the axonal terminal component of the response is not influenced by the preceding interspike interval, the postsynaptic component clearly is. Figure $4 d$ shows, for this same CTect neuron, the relationship between the preceding interspike interval and the magnitude of the axonal (left) and postsynaptic current sinks (right) as a function of depth on the 16-channel probe. As shown in Figure $4 c$, whereas the axon terminal potential is not affected by this variable, the postsynaptic current sinks increases with the duration of the preceding interspike interval.

Qualitatively similar results were obtained in each of 21 additional cases in which a sufficient number of spontaneous spikes were collected. CTect neurons generally fire at rates of $4-8 / \mathrm{s}$, and large data sets are required to insure that a sufficient number of spikes occur at each of the preceding intervals to be tested (mean value for total spike trains, $\sim 17,000$ spikes for the 21 cases studied). In each of these 21 cases, we required at least 500 spikes to occur at each of the intervals tested, and to generate reliable postsynaptic responses (as defined in Materials and Methods) at each intervals tested. Because of these stringent criteria, CTect neurons generating weak postsynaptic responses $(<2.4 \mu \mathrm{V}$; based on the entire CTect spike train) were not used for these tests.

In each of these 21 cases, CTect impulses with longer preceding interspike intervals generated postsynaptic current sinks of greater magnitude than did impulses preceded by short interspike intervals. However, the magnitude of the enhanced response varied considerably among different CTect neurons. Figure $5 a$ shows, for each of these 21 CTect neurons, the magnitude of the postsynaptic current sinks that were generated by impulses with various preceding interspike intervals. We plotted the amplitude of the current sink occurring only during the initial $1 \mathrm{~ms}$ of the postsynaptic response to avoid the possibility of including disynaptic currents. For each CTect neuron, the values generated are normalized against the value obtained at the shortest intervals (5-25 ms). For some CTect neurons, current sinks generated by impulses with the longest preceding intervals ( $>450 \mathrm{~ms}$ ) were two to three times greater than impulses preceded by the shortest intervals, but other neurons showed only a mild enhancement. Thus, all of the CTect neurons show enhanced postsynaptic responses at the longer intervals, but there are clear differences among them in the magnitude of this effect. Figure $5 b$ shows that, for the 21 CTect neurons shown in Figure $5 a$, there was a strong correlation between the degree of enhancement generated at the two longest intervals $(250-450$ and $>450 \mathrm{~ms} ; r=+0.83$; 
a

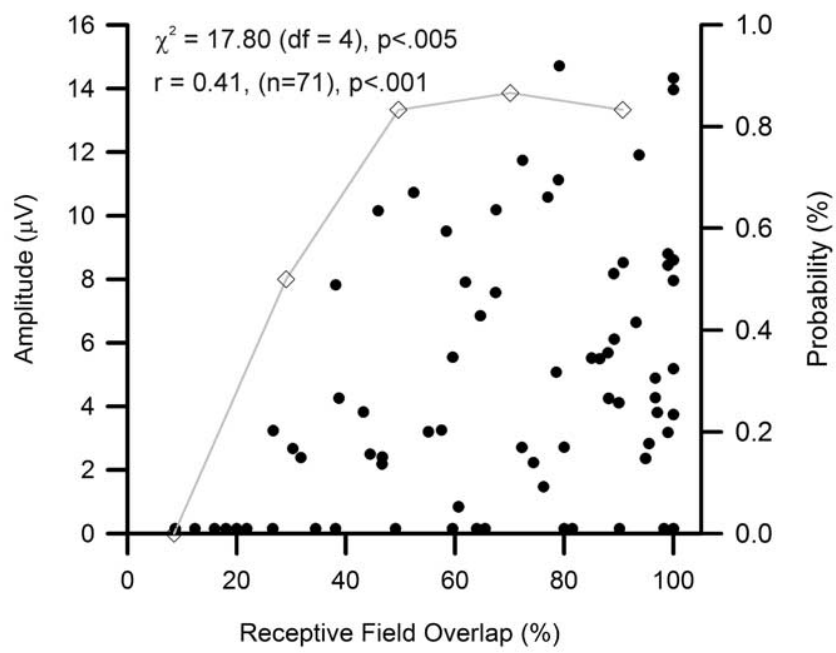

b

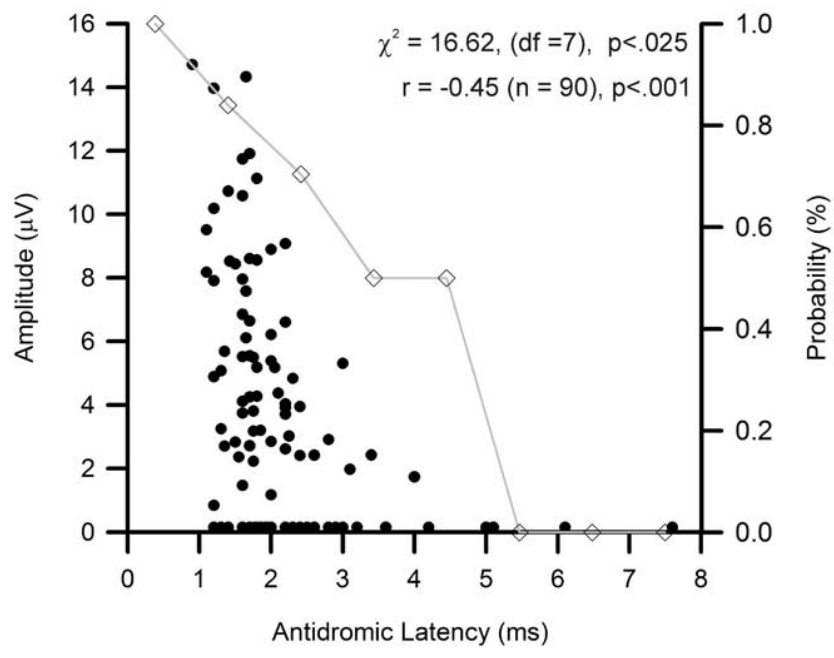

Figure 3. The influence of retinotopic alignment (a) and conduction time along the CTect axon ( $\boldsymbol{b}$ ) on the amplitude (solid points) and probability (open diamonds) of the postsynaptic impact. Because slowly conducting axons provide a weak synaptic impact, regardless of receptive field overlap (see $\boldsymbol{b}$ ), only fast-conducting (Tect neurons are presented in $\boldsymbol{a}$. Similarly, in $\boldsymbol{b}$, only cases with at least $50 \%$ receptive field overlap are presented.

$p \leq 0.0001$ ), indicating that these differences among CTect neurons were reliable.

We had intended to examine the synaptic dynamics of CTect "burst" responses but found too few such events in our spontaneously firing CTect neurons to allow reliable generation of spike-triggered field activity. Under our experimental conditions, most $(>80 \%)$ of our CTect neurons generated very few $(<1 \%)$ interspike intervals of $<4 \mathrm{~ms}$. Very few bursts were seen even when subjects were in an inattentive/drowsy state [during hippocampal high-voltage, irregular activity (HVIR)], and thalamic neurons were generating many burst responses (see supplemental Fig. 1, available at www.jneurosci.org as supplemental material). Some of these neurons did show burst-like responses to periodic visual stimulation, but such stimulation generated large-amplitude field potentials within the SC that made it impossible to detect the much smaller currents that were generated by impulses of single CTect neurons.

We also asked whether the postsynaptic impact of a CTect impulse differed in the alert versus inattentive/drowsy state. To do this, we divided the data files of 16 CTect neurons into periods in which the hippocampal EEG was dominated by theta activity, and periods dominated by HVIR. For this test, neurons were selected from the pool of 21 neurons used to study synaptic dynamics (above). Five of these 21 neurons could not be used in the present analysis, because hippocampal EEG activity was not of sufficient clarity to segregate the data files into unambiguous periods of "theta" versus "HVIR." We have previously shown that thalamic neurons are in "relay mode" during hippocampal theta activity but that thalamic spike trains become irregular and "bursty" during HVIR (Swadlow and Gusev, 2001; Bezdudnaya et al., 2006). Notably, we found no significant relationship between the hippocampal EEG and either the frequency of spontaneous CTect impulses [mean firing rates, 5.4 spikes (sp)/s during theta and $6.1 \mathrm{sp} / \mathrm{s}$ during HVIR] or the synaptic impact of CTect impulses (with either long or short preceding interspike intervals) on the SC (Fig. $5 c$ ).

\section{Discussion}

The impact of a CTect impulse on the SC

Synaptic responses generated by single thalamocortical and corticocortical neurons have been subject to intensive in vitro (Thomson et al., 1993; Stratford et al., 1996; Gil et al., 1997, 1999; Kozloski et al., 2001; Feldmeyer et al., 2002) and in vivo analyses (Alonso et al., 2001; Martinez and Alonso, 2001; Swadlow and Gusev, 2001). There have been no studies, however, of the synaptic impact of single CTect neurons within the SC. Nor have there been morphological studies of terminal arborizations of single CTect axons. Our results, therefore, provide an initial view of the synaptic impact of single CTect neurons through the depths of the SC. We show that CTect neurons provide precise and potent excitatory synaptic impact. A CTect impulse generates a distinct axon terminal potential, with a latency closely matching the antidromic conduction time. This axonal potential is followed by a fast-rising inward current, at an interval consistent with a fast, ionotropic-mediated EPSP. CTect synapses are glutamatergic (Jeon et al., 1997), and NMDA receptors play a role in mediating the influence of CTect input to the SC (Binns and Salt, 1996). However, monosynaptic currents generated by CTect impulses demonstrated short synaptic delays and rapid rise times (Fig. 1), suggesting that underlying receptor mechanisms for these currents are probably AMPA/kainate (Edmonds et al., 1995).

\section{Do presynaptic and postsynaptic currents reflect the impact} of single CTect neurons?

Several factors argue that synchronously firing CTect neurons did not contribute significantly to our results: (1) early components of spike-triggered SC profiles display high temporal precision (rise times of axonal and monosynaptic potentials, 0.17 and $0.47 \mathrm{~ms}$, respectively). In contrast, even "precisely correlated firing" among dorsal lateral geniculate nucleus (LGNd) neurons (Alonso et al., 1996) and "sharp synchrony" among fast-spike cortical interneurons (Swadlow et al., 1998) are relatively slower ( $\pm 1 \mathrm{~ms})$. Thus, the time course of these rapid events would have blurred considerably if synchronous CTect activity contributed 
significantly. (2) Additional temporal blurring of axonal and postsynaptic responses would result from the wide range of conduction times along CTect axons (Figs. 1d, 3b). (3) Neighboring CTect neurons displayed distinct axonal and postsynaptic laminar profiles (Figs. $1,2)$, and synchronous CTect firing would have blurred these profiles. (4) Finally, we examined 32 pairs of neighboring CTect neurons (our unpublished observations) and saw no precisely correlated firing.

\section{Retinotopic precision and conduction velocity of the CTect axon}

Both the amplitude and the probability of postsynaptic CTect impacts were strongly related to degree of retinotopic overlap. This result is consistent with morphological and physiological (electrical stimulation) studies indicating a retinotopic projection from V1 to SC (Mcllwain, 1973; Hollander and Schonitzer, 1983; Hofbauer and Hollander, 1986; Harting et al., 1992). Axonal conduction time was another powerful predictor of postsynaptic impact within the SC. Whereas $>80 \%$ of retinotopically aligned fast CTect axons (conduction times of $<2 \mathrm{~ms}$ ) provided reliable synaptic drive to the aligned region of SC, none of four CTect axons with conduction times of $>5 \mathrm{~ms}$ did so (Fig. $3 b$ ). Slowly conducting CTect axons may represent fine branches of CTect axons that project more extensively to other subcortical structures, and such branches may support fewer terminal arbors than thicker axons.

\section{Depth of monosynaptic current sinks generated by single CTect neurons}

Multiple cortical areas are known to project to the SC in a laminar-specific manner (Harting et al., 1992; Pare and Wurtz, 1997; Helminski and Segraves, 2003). Synaptic currents generated by most of our V1 CTect neurons reached peak values $500-700 \mu \mathrm{m}$ beneath the surface of the SC. This depth is consistent with a dense band of V1 CTect terminals $\sim 500 \mu \mathrm{m}$ beneath the surface of the SC (Hollander and Schonitzer, 1983), just beneath the focus of retinal input (Hofbauer and Hollander, 1986). However, Hollander and Schonitzer (1983) also reported a secondary band of

CTect terminals $\sim 200 \mu \mathrm{m}$ beneath the surface, but we detected no evidence of this superficial input. It may be that our single-axon CSD method is sensitive only to retinotopically focused and powerful synaptic inputs generated by fastconducting CTect neurons. Indeed, it is important to recog-
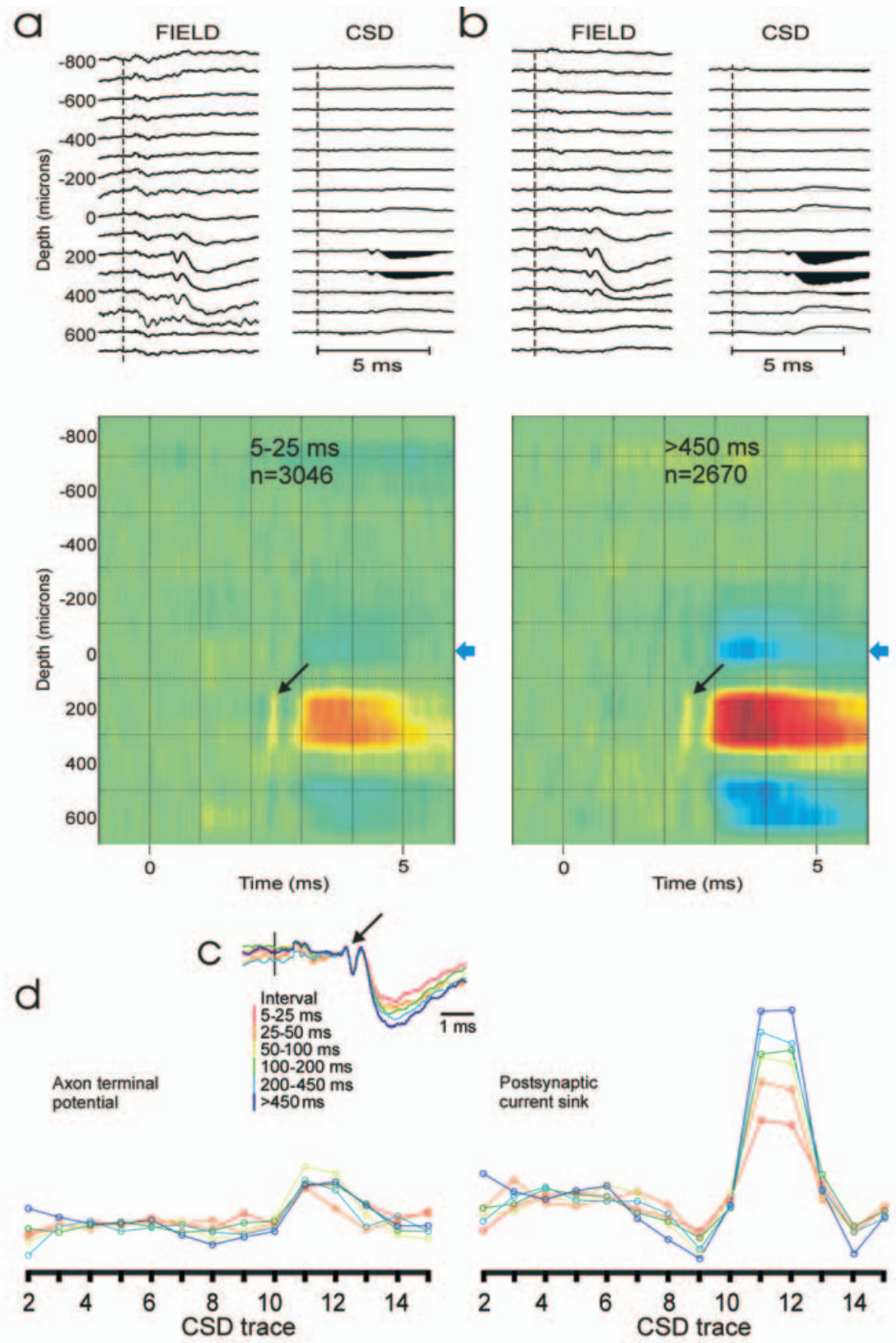

Figure 4. Spike-triggered CSD depth profiles of a CTect neuron that generated a powerful current sink in the superficial SC. $\boldsymbol{a}$, A total of 3046 spikes with short (5-25 ms) preceding interspike intervals was used to generate the field potentials (top left) and CSD (top right; colorized version below) depth profiles. $\boldsymbol{b}$, The field and CSD depth profiles for this same CTect neuron, but these were generated by 2670 impulses with long preceding interspike intervals ( $>450 \mathrm{~ms}$ ). The gain settings and color intensities for the field and CSD profiles in $\boldsymbol{a}$ and $\boldsymbol{b}$ are identical, and the oblique arrows indicate the axon terminal potentials. $\boldsymbol{c}$, The spiketriggered field potentials that were recorded on channel 11 of our 16-channel probe. Different color traces were based on spikes with differing preceding interspike intervals. Whereas the axonal terminal component of the response (arrow) is not influenced by the preceding interspike interval, the postsynaptic component clearly is. $\boldsymbol{d}$, For this same CTect neuron, the relationship between the preceding interspike interval and the magnitude of the axonal (left) and postsynaptic current sinks (right) as a function of depth on the 16 channel probe. We measured the peak-peak amplitude of the axon terminal potential. For measures of the postsynaptic current sink, we measured the amplitude of the current sink that occurred during the initial $1 \mathrm{~ms}$ of the postsynaptic response to avoid the possibility of including disynaptic currents.

nize that CSD analyses provide estimates of net current flow at a given depth. Strong net current sinks will reflect sites of relatively strong excitatory synaptic drive. However, sites receiving weak excitatory synaptic drive but displaying larger passive outward currents will be seen as net current sources. 
a

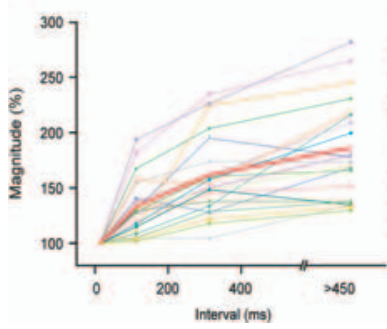

b

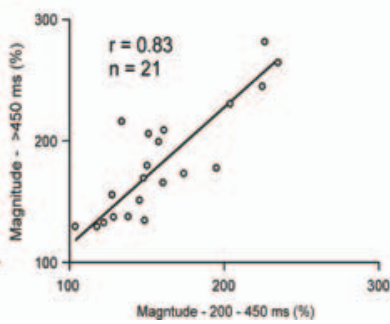

C

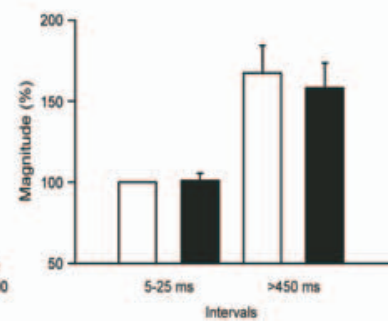

Figure 5. $\boldsymbol{a}$, The magnitude of the postsynaptic current sink generated by impulses with various preceding interspike intervals, for 21 well studied CTect neurons. The magnitude of the response is given on the $y$-axis as a percentage of the response for "short" $(5-25 \mathrm{~ms})$ preceding interspike intervals. As in Figure 4, the value is that of the peak response during the initial $1 \mathrm{~ms}$ of the postsynaptic response. The red line denotes the mean values. $\boldsymbol{b}$, For these 21 CTect neurons, the relationship between the postsynaptic enhancement that was generated at the two longest intervals ( $250-450$ and $>450 \mathrm{~ms} ; r=+0.83, p=<$ 0.0001). c, Lack of state dependence in the magnitude and dynamics of (Tect synaptic impact in the alert (white) or inattentive/ drowsy state (black). For each of the 16 cells studied, the postsynaptic response amplitude was normalized against the value obtained in the alert state, at short (5-25 ms) preceding interspike intervals (shown as a value of 100\%). Error bars indicate SE.

This may sharpen the depth gradient of a current sink with respect to the underlying distribution of active synaptic sites.

\section{CTect synaptic dynamics within the SC: effects of preceding interval and state}

Our results showing a powerful effect of preceding interspike interval on the amplitude of postsynaptic (but not axonal) responses indicate a chronic depression at awake CTect synapses, in which long preceding intervals allow recovery from depression and result in maximum postsynaptic responses (Ramcharan et al., 2000; Swadlow and Gusev, 2001; Castro-Alamancos, 2002; Swadlow et al., 2002; Boudreau and Ferster, 2005). These results are remarkably similar to recent studies of thalamocortical synapses in awake rabbits (Swadlow and Gusev, 2001; Swadlow et al., 2002). Although dynamics of CTect synapses within the SC have not been previously examined, intracortical synapses of the collaterals of CTect axons exhibit target-specific depression/facilitation (Kozloski et al., 2001).

CTect neurons showed marked differences in the degree of depression (Fig. $5 b, c$ ), similar to such differences seen among thalamocortical synaptic contacts studied in vitro (Gil et al., 1997) (Fig. 2c). Of course, the degree of depression shown here reflects an average depression for all synapses activated by CTect impulses. Because synaptic depression/facilitation can be target specific (Markram et al., 1998; Reyes et al., 1998; Kozloski et al., 2001), differences among CTect cells could reflect differences in their SC target populations. Alternatively, some CTect axons may generate more depressing synaptic contacts on similar synaptic targets.

We found no EEG state dependence in the amplitude or dynamics of postsynaptic CTect impact. Notably, few burst-like events were seen in CTect neurons, even during hippocampal HVIR, when subjects are inattentive and thalamus is bursty. This was unexpected, because nearly all CTect neurons studied in vitro have intrinsically bursting membrane properties (Kasper et al., 1994; Rumberger et al., 1998; Tsiola et al., 2003). Possibly, burstlike responses in CTect neurons are limited to sleep states (Wang and McCormick, 1993) and/or stimulus conditions that generate excitatory inputs after prolonged inhibition.

\section{What is the functional role of CTect input to SC?}

It has long been known that inactivation of $\mathrm{V} 1$ causes reduced responsiveness in many superficial SC neurons, and that some receptive field properties are lost or modified (e.g., directional selectivity, velocity tuning, binocularity) (Rizzolatti et al., 1970; Rosenquist and Palmer, 1971; Ogasawara et al., 1984). However, the mechanisms of this influence are still unknown (Hashemi-Nezhad et al., 2003). It is not known, for example, whether these response properties are conveyed to SC neurons directly by "driving" (Sherman and Guillery, 1998) CTect input (as retinal axons convey receptive fields to LGNd neurons) (Usrey et al., 1999), whether the CTect influence is modulatory (consistent with demonstrated NMDA contributions) (Binns and Salt, 1996), or even whether these effects result from direct monosynaptic CTect input. Our results suggest that single CTect neurons probably serve as drivers of postsynaptic targets within the SC and could directly mediate some of the above effects. Thus, the rise time, time course, and amplitude of observed monosynaptic currents were very similar to those generated within somatosensory and visual cortices (Swadlow and Gusev, 2000; Swadlow et al., 2002; Bereshpolova et al., 2005; Jin et al., 2005) by thalamic afferents that potently drive cortical neurons (Gil et al., 1997, 1999; Stratford et al., 1996; Alonso et al., 2001; Swadlow and Gusev, 2001). Single thalamocortical neurons contribute several thousand synapses within a small, topographically aligned region of cortex (Freund et al., 1985; Humphrey et al., 1985; Jensen and Killackey, 1987). Terminal arborizations of single CTect axons have not been examined, but the similarity of our results to the above studies of single thalamocortical axons suggest that single CTect neurons may contribute a similarly dense distribution of boutons within the aligned region of superficial SC. Moreover, the evidence for activity-dependent synaptic depression obtained here is similar to that seen at thalamocortical synapses, both in vitro (Castro-Alamancos and Connors, 1996; Gil et al., 1997) and in vivo (Swadlow and Gusev, 2001; Swadlow et al., 2002; Boudreau and Ferster, 2005), suggesting a similar high probability of transmitter release (Dobrunz and Stevens, 1997).

The above considerations raise questions concerning cell classes in superficial SC that receive CTect input (Mooney et al., 1988), the specificity of CTect input to these SC recipient neurons (Alonso et al., 2001; Swadlow and Gusev, 2002), and the manner in which input from single CTect neurons interacts with retinal input to recipient cells (McIlwain, 1977; Berson, 1988). These questions may be addressed by applying extracellular crosscorrelation methods (Alonso et al., 2001; Swadlow and Gusev, 2001) to the spike trains of CTect neurons and SC neurons of different classes (identified by antidromic or other means) within retinotopically aligned regions of the superficial SC.

\section{References}

Alonso JM, Usrey WM, Reid RC (1996) Precisely correlated firing in cells of the lateral geniculate nucleus. Nature 383:815-819.

Alonso JM, Usrey WM, Reid RC (2001) Rules of connectivity between geniculate cells and simple cells in cat primary visual cortex. J Neurosci 21:4002-4015.

Bereshpolova YI, Stoelzel CR, Gusev AG, Swadlow HA (2005) Retinotopic precision and dynamics of the awake geniculocortical synapse. Soc Neurosci Abstr 31:742.8. 
Berson D (1988) Convergence of retinal w-cell and corticotectal input to cells of the cat superior colliculus. J Neurophysiol 60:1861-1873.

Bezdudnaya T, Cano M, Bereshpolova Y, Stoelzel CR, Alonso JM, Swadlow HA (2006) Thalamic burst mode and inattention in the awak LGNd. Neuron 49:421-432.

Binns KE, Salt TE (1996) Corticofugal influences on visual responses in cat superior colliculus: the role of NMDA receptors. Vis Neurosci 13:683-694.

Borroni AM, Vaknin G, Berry R, Teyler TJ (1991) Methods for studying the conductance changes associated with synaptic activation of forebrain slices: the interpretation of field potentials using CSD profiles. J Neurosci Methods 39:89-102.

Boudreau CE, Ferster D (2005) Short-term depression in thalamocortical synapses of cat primary visual cortex. J Neurosci 31:7179-7190.

Buzsaki G, Kandel A (1998) Somadendritic backpropagation of action potentials in cortical pyramidal cells of the awake rat. J Neurophysiol 79:1587-1591.

Castro-Alamancos MA (2002) Role of thalamocortical sensory suppression during arousal: focusing sensory inputs in neocortex. J Neurosci 22:9651-9655.

Castro-Alamancos MA, Connors BW (1996) Spatiotemporal properties of short-term plasticity in sensorimotor thalamocortical pathways of the rat. J Neurosci 16:2767-2779.

Chagnac-Amitai Y, Connors BW (1989) Synchronized excitation and inhibition driven by intrinsically bursting neurons in neocortex. J Neurophysiol 62:1149-1162.

Collewijn H (1977) Eye- and head movements in freely moving rabbits. J Physiol (Lond) 266:471-498.

Connors BW, Gutnick MJ, Prince DA (1982) Electrophysiological properties of neocortical neurons in vitro. J Neurophysiol 48:1302-1320.

Dobrunz LE, Stevens CF (1997) Heterogeneity of release probability, facilitation, and depletion at central synapses. Neuron 18:995-1008.

Edmonds B, Gibb AJ, Colquhoun D (1995) Mechanisms of activation of glutamate receptors and the time course of excitatory synaptic currents. Annu Rev Physiol 57:495-519.

Feldmeyer D, Lubke J, Angus Silver R, Sakmann B (2002) Synaptic connections between layer 4 spiny neurone-layer $2 / 3$ pyramidal cell pairs in juvenile rat barrel cortex: physiology and anatomy of interlaminar signaling within a cortical column. J Physiol (Lond) 583:803-822.

Freeman JA, Nicholson C (1975) Experimental optimization of current source-density technique for anuran cerebellum. J Neurophysiol 38:369-382.

Freund TF, Martin KA, Whitteridge D (1985) Innervation of cat visual areas 17 and 18 by physiologically identified X-and Y-type thalamic afferents. I. Arborization patterns and quantitative distribution of postsynaptic elements. J Comp Neurol 242:263-274.

Gil Z, Connors BW, Amitai Y (1997) Differential regulation of neocortical synapses by neuromodulators and activity. Neuron 19:679-686.

Gil Z, Connors BW, Amitai Y (1999) Efficacy of thalamocortical and intracortical synaptic connections: quanta, innervation, and reliability. Neuron 23:385-397.

Haberly LB, Shepherd GM (1973) Current-density analysis of summed evoked potentials in opossum prepyriform cortex. J Neurophysiol 36:789-803.

Hallman LE, Schofield BR, Lin CS (1988) Dendritic morphology and axon collaterals of corticotectal, corticopontine, and callosal neurons in layer 5 of primary visual cortex of the hooded rat. J Comp Neurol 272:149-160.

Harting JK, Updyke BV, Van Lieshout DP (1992) Corticotectal projections in the cat: anterograde transport studies of twenty-five cortical areas. J Comp Neurol 324:379-414.

Hashemi-Nezhad M, Wang C, Burke W, Dreher B (2003) Area 21a of cat visual cortex strongly modulates neuronal activities in the superior colliculus. J Physiol (Lond) 550:535-552.

Helminski JO, Segraves MA (2003) Macaque frontal eye field input to saccade-related neurons in the superior colliculus. J Neurophysiol 90:1046-1962.

Hofbauer A, Hollander H (1986) . Synaptic connections of cortical and retinal terminals in the superior colliculus of the rabbit: an electron microscopic double labeling study. Exp Brain Res 65:145-155.

Hollander H, Schonitzer K (1983) Corticotectal terminals in the superior colliculus of the rabbit: a light- and electron microscopic analysis using horseradish peroxidase (HRP)-tetramethylbenzidene (TMB). J Comp Neurol 219:81-87.

Hubner M, Schwarz C, Bolz J (1990) Morphological types of projection neurons in layer 5 of cat visual cortex. J Comp Neurol 301:655-674.

Humphrey AL, Sur M, Uhlrich DJ, Sherman SM (1985) Projection patterns of individual $\mathrm{X}$ - and $\mathrm{Y}$-cell axons from the lateral geniculate nucleus to cortical area 17 in the cat. J Comp Neurol 233:159-189.

Jensen KF, Killackey HP (1987) Terminal arbors of axons projecting to the somatosensory cortex of the adult rat. I. The normal morphology of specific thalamocortical afferents. J Neurosci 7:3529-3543.

Jeon CJ, Hartman MK, Mize RR (1997) Glutamate-like immunoreactivity in the cat superior colliculus and visual cortex: further evidence that glutamate is the neurotransmitter of the corticocollicular pathway. Vis Neurosci 14:27-37.

Jin JZ, Weng C, Yeh CI, Swadlow HA, Alonso JM (2005) Synaptic impact of single geniculate afferents through the depth of cat primary visual cortex. Soc Neurosci Abstr 31:742.7.

Jones JP, Palmer LA (1987) The two-dimensional spatial structure of simple receptive fields in cat striate cortex. J Neurophysiol 58:1187-1211.

Kasper EM, Larkman AU, Lubke J, Blakemore C (1994) Pyramidal neurons in layer 5 of the rat visual cortex. I. Correlation among cell morphology, intrinsic electrophysiological properties, and axon targets. J Comp Neurol 339:459-474.

Keizer K, Kuypers HG, Ronday HK (1987) Branching cortical neurons in cat which project to the colliculi and to the pons: a retrograde fluorescent double-labeling study. Exp Brain Res 67:1-15.

Kirkwood PA (1995) Synaptic excitation in the thoracic spinal cord from expiratory bulbospinal neurons in the cat. J Physiol (Lond) 484:201-225.

Kozloski J, Hamzei-Sichani F, Yuste R (2001) Stereotyped position of local synaptic targets in neocortex. Science 293:868-872.

Levick RW (1967) Receptive fields and trigger features of ganglion cells in the visual streak of the rabbits retina. J Physiol (Lond) 188:285-307.

Markram H, Wang Y, Tsodyks M (1998) Differential signaling via the same axon of neocortical pyramidal neurons. Proc Natl Acad Sci USA 95:5323-5328.

Martinez LM, Alonso J-M (2001) Construction of complex receptive fields in cat primary visual cortex. Neuron 32:515-525.

McCormick DA, Connors BW, Lighthall JW, Prince DA (1985) Comparative electrophysiology of pyramidal and sparsely spiny stellate neurons of the neocortex. J Neurophysiol 54:782-806.

McIlwain JT (1973) Topographic relationships in projection from striate cortex to superior colliculus of the cat. J Neurophysiol 36:690-701.

McIlwain JT (1977) Topographic organization and convergence in corticotectal projections from areas 17, 18, and 19 in the cat. J Neurophysiol 40:189-198.

Mooney RD, Nikoletseas MM, Ruiz SA, Rhoades RW (1988) Receptivefield properties and morphological characteristics of the superior collicular neurons that project to the lateral posterior and dorsal lateral geniculate nuclei in the hamster. J Neurophysiol 59:1333-1351.

Ogasawara JG, McHaffie JG, Stein BE (1984) Two visual corticotectal systems in cat. J Neurophysiol 52:1226-1245.

Pare M, Wurtz RH (1997) Monkey posterior parietal cortex neurons antidromically activated from superior colliculus. J Neurophysiol 78:3493-3497.

Ramcharan EJ, Cox CL, Zhan XJ, Sherman SM, Gnadt JW (2000) Cellular mechanisms underlying activity patterns in the monkey thalamus during visual behavior. J Neurophysiol 84:1982-1987.

Reitboeck HJ (1983) Fiber microelectrodes for electrophysiological recordings. J Neurosci Methods 8:249-262.

Reyes A, Lujan R, Rozov A, Burnashev N, Sakmann B (1998) Target-cell specific facilitation and depression in neocortical circuits. Nat Neurosci 1:279-285.

Rizzolatti G, Tradardi V, Camarda R (1970) Unit responses to visual stimuli in the cat's superior colliculus after removal of the visual cortex. Brain Res 24:336-339.

Rosenquist AC, Palmer LA (1971) Visual receptive field properties of cells of the superior colliculus after cortical lesions in the cat. Exp Neurol 33:629-652.

Rumberger A, Schmidt M, Lohmann H, Hoffman K-P (1998) Correlation of electrophysiology, morphology, and functions in corticotectal and corticopretectal projection neurons in rat visual cortex. Exp Brain Res 119:375-390. 
Sherman SM, Guillery RW (1998) On the actions that one nerve cell can have on another: distinguishing "drivers" from "modulators." Proc Natl Acad Sci USA 95:121-126.

Stratford KJ, Tarczy-Hormoch K, Martin KAC, Bannister NJ, Jack JJB (1996) Excitatory synaptic inputs to spiny stellate cells in cat visual cortex. Nature 382:258-261.

Swadlow HA (1995) Influence of VPM afferents on putative inhibitory interneurons in S1 of the awake rabbit: evidence from cross-correlation, microstimulation, and latencies to peripheral sensory stimulation. J Neurophysiol 73:1584-1599.

Swadlow HA, Gusev AG (2000) The influence of single VB thalamocortical impulses on barrel columns of rabbit somatosensory cortex. J Neurophysiol 83:2802-2813.

Swadlow HA, Gusev AG (2001) The impact of "bursting" thalamic impulses on a neocortical synapse. Nat Neurosci 4:402-408.

Swadlow HA, Gusev AG (2002) Receptive field construction in cortical inhibitory interneurons. Nat Neurosci 5:403-404.

Swadlow HA, Weyand TG (1981) Efferent systems of the rabbit visual cortex: laminar distribution of the cells of origin, axonal conduction velocities, and identification of axonal branches. J Comp Neurol 203:799-822.

Swadlow HA, Weyand TG (1987) Corticogeniculate neurons, corticotectal neurons and suspected interneurons in visual cortex of the awake rabbit: receptive field properties, axonal properties and effects of EEG arousal. J Neurophysiol 57:977-1001.

Swadlow HA, Beloozerova I, Sirota M (1998) Sharp, local synchrony among putative feed-forward inhibitory interneurons of rabbit somatosensory cortex. J Neurophysiol 79:567-582.

Swadlow HA, Gusev AG, Bezdudnaya T (2002) Activation of a cortical column by a thalamocortical impulse. J Neurosci 22:7766-7773.

Thomson AM, Deuchars J, West DC (1993) Large, deep-layer pyramidalpyramidal single axon EPSPs in slices of rat motor cortex display paired pulse and frequency-dependent depression, mediated presynaptically and self-facilitating, mediated postsynaptically. J Neurophysiol 70:2354-2369.

Tsiola A, Hamzei-Sichani F, Peterlin Z, Yuste R (2003) Quantitative morphologic classification of layer 5 neurons from mouse primary visual cortex. J Comp Neurol 461:415-428.

Usrey WM, Reppas JB, Reid RC (1999) Specificity and strength of retinogeniculate connections. J Neurophysiol 82:3527-3540.

Wang Z, McCormick DA (1993) Control of firing mode of corticotectal and corticopontine layer $\mathrm{V}$ burst-generating neurons by norepinephrine, acetylcholine, and 1S,3R-ACPD. J Neurosci 13:2199-2216.

White EL (1990) Cortical circuits. Boston: Birkhauser. 